\title{
Label-free quantitative proteomic analysis reveals potential biomarkers for early healing in cutaneous leishmaniasis
}

\author{
Andrés Montoya ${ }^{1}{ }^{\text {, }}$ Manuel C López ${ }^{2}{ }^{\text {, Ivan D. Vélez }}{ }^{1}$ ， Sara M. Robledo ${ }^{\text {Corresp. } 1}$ \\ 1 PECET, Facultad de Medicina, Universidad de Antioquia, Medellin, Antioquia, Colombia \\ 2 Molecular Biology Departmentm Consejo Superior de Investigaciones Científicas, Instituto de Parasitología y Biomedicina "López Neyra", Medellín, \\ (State), Colombia \\ Corresponding Author: Sara M. Robledo \\ Email address: sara.robledo@udea.edu.co
}

Background. Leishmaniasis is a parasitic disease caused by more than 20 species of the Leishmania genus. The disease is globally distributed and is endemic in 97 countries and three territories in the tropical and subtropical regions. The efficacy of the current treatments is becoming increasingly low either due to incomplete treatment or resistant parasites. Failure of treatment is frequent, and therefore, the search for early biomarkers of therapeutic response in cutaneous leishmaniasis $(C L)$ is urgently needed. Objective: The aim of this study was to compare the proteomic profiles in patients with CL before and after 7 days of treatment and identify early biomarkers of curative response. Methods. Four patients with a parasitological diagnosis of leishmaniasis with confirmation of species by PCR-RFLP were recruited. All patients had a single lesion, and a protein from the middle of the ulcer was quantified by liquid chromatography and mass spectrometry (LC-MS).

Results. Twelve proteins showed differential expression in the comparative LCelectrospray ionization MS/MS (LC-ESI-MS/MS) triplicate analysis. Seven of them were upregulated and five of them were down-regulated. Calcium binding proteins $A 2, A 8$, and $A 9$ and hemoglobin subunits alpha-2 and delta showed high correlation with epidermis development and immune response. Conclusion. We identified changes in the profiles of proteins that had a positive therapeutic response to the treatment. The proteins identified with differential expression are related to the reduction of inflammation and increased tissue repair. These proteins can be useful as biomarkers for early monitoring of therapeutic response in cutaneous leishmaniasis. 


\section{Label-free quantitative proteomic analysis reveals}

potential biomarkers for early healing in cutaneous leishmaniasis

Andrés Montoya ${ }^{1}$, Manuel Carlos López $^{2}$, Iván D. Vélez ${ }^{1}$, Sara M. Robledo ${ }^{1}$ (1)

1. PECET, Facultad de Medicina, Universidad de Antioquia, Medellín, Colombia

2. Instituto de Parasitología y Biomedicina "López Neyra", Molecular Biology Department.

Consejo Superior de Investigaciones Científicas, Granada, Spain.

Short tittle: Biomarkers in cutaneous leishmaniasis

Corresponding Author:

Sara M. Robledo ${ }^{1}$

Calle 70 \# 52-21. Medellín, Colombia. Postal code 0500100. Email: sara.robledo@udea.edu.co

\section{ABSTRACT}

Background. Leishmaniasis is a parasitic disease caused by more than 20 species of the Leishmania genus. The disease is globally distributed and is endemic in 97 countries and three territories in the tropical and subtropical regions. The efficacy of the current treatments is becoming increasingly low either due to incomplete treatment or resistant parasites. Failure of treatment is frequent, and therefore, the search for early biomarkers of therapeutic response in cutaneous leishmaniasis (CL) is urgently needed.

Objective: The aim of this study was to compare the proteomic profiles in patients with CL before and after 7 days of treatment and identify early biomarkers of curative response.

Methods. Four patients with a parasitological diagnosis of leishmaniasis with confirmation of species by PCR-RFLP were recruited. All patients had a single lesion, and a protein from the middle of the ulcer was quantified by liquid chromatography and mass spectrometry (LC-MS). 
30 Results. Twelve proteins showed differential expression in the comparative LC-electrospray 31 ionization MS/MS (LC-ESI-MS/MS) triplicate analysis. Seven of them were up-regulated and 32 five of them were down-regulated. Calcium binding proteins A2, A8, and A9 and hemoglobin 33 subunits alpha-2 and delta showed high correlation with epidermis development and immune 34 response.

35 Conclusion. We identified changes in the profiles of proteins that had a positive therapeutic 36 response to the treatment. The proteins identified with differential expression are related to the 37 reduction of inflammation and increased tissue repair. These proteins can be useful as 38 biomarkers for early monitoring of therapeutic response in cutaneous leishmaniasis.

\section{INTRODUCTION}

41 Leishmaniasis is a parasitic disease caused by more than 20 species of Leishmania genus. The 42 parasite is transmitted by Diptera insects of the genus Phlebotomus (Old World) and Lutzomyia 43 (New World) (1). The disease is distributed on five continents and is endemic to 97 countries and 443 territories in the tropical and subtropical regions (2). Cutaneous leishmaniasis (CL), which is 45 the most prevalent clinical form, manifests as a single or multiple ulcer with indurated edges, is 46 painless, and presents a granular background that may or may not be covered by an exudate; the

Despite the high number of people affected, leishmaniasis control programs present difficulties not only with the diagnosis of cases and the control of vectors to reduce transmission but also with the treatment, given that the therapeutic response with the available drugs is becoming poorer due to incomplete treatments or the development of resistance in the parasites (4). Unfortunately, the confirmation of a definitive cure is complicated by the possibility of relapses after an initial cure, making necessary long follow-up periods (up to 6 or 12 months).

55

The prognosis of the treatment can be improved by detecting an early curative response or treatment failure. Such markers should also specifically correlate with the response to treatment and predict the long-term clinical outcome, by non-invasive sampling methods. 
$60 \mathrm{Up}$ to now, the main advances have been made in visceral leishmaniasis (VL) where levels of

61 IgG1 and IgG2 antibodies, TNF $\alpha$, IL10, and parasite load were proposed as biomarkers of

62 infection (5-7). IFN- $\gamma$, IL-2, monokine-induced-by-IFN- $\gamma$ (MIG) and IFN- $\gamma$-inducible protein-10

63 (IP-10) could be used as markers of cure in immunocompetent patients with VL caused by $L$.

64 infantum (8). In case of CL, the levels of IL4, IL8, INF $\gamma$, and MMP2 in skin biopsies and levels

65 of TNF $\alpha$, IL10, IL15, IL32 $\gamma$, TGF $\beta$, sCD26, sCD30, Cortisol, Prolactin, and SOD1 in serum,

66 have been associated with infection and disease susceptibility (9-11). Nevertheless, these

67

68

69

70

71

72

73

74

75

76

77

78

79

80

81

82

83

84

85

86

87

88

89

90

candidates are not fully validated as biomarkers of infection, and other biomarkers for treatment monitoring have not been identified or validated.

Analysis by liquid chromatography and electrospray ionization in tandem mass spectrometry (LC-ESI-MS/MS) has emerged as one of the most robust alternatives for the comparison of proteomic profiles through a quantitative approach based on labels (12). Quantitative analysis has been useful in clinical proteomics, proteomics of subcellular protein and high-resolution comparative proteomics (13), which has advanced the analysis of systems biology, particularly in the elucidation of the mechanisms that underlie the pathophysiology of several diseases.

The introduction of stable isotopes is the basis for the quantification in label-based approach and various protocols are now available that vary depending on the used methods for the incorporation of isotopes in the peptides/proteins. One of these methods is the isobaric tag for relative and absolute quantification (iTRAQ). The iTRAQ is a label-based method that allow the simultaneous analysis of eight samples in a single run of MS (multiplexing), resulting in reduced analytical variability. (14). With this method, the labeled peptides are shown as a single peak in the MS spectrum and, after fragmentation of peptides in the MS/MS analysis, the isotopes are released and peptides are identified according to their masses. This type of proteomic analysis results in data with high quality, sensitivity and a wide dynamic range (15).

7 The use of unlabeled protein profiles to examine differentially expressed proteins in tissue samples largely eliminates the variations and biases in replicate MS measurements and has allowed for the exploration of the mechanism of tumorigenesis and the discovery of biomarkers in various diseases $(16,17)$. 
91

92 Based on the advantages offered by this methodology, the aim of this study was to find and validate early biomarkers of therapeutic response in CL using proteomics without labeling in order to provide patients with adequate monitoring and identify early treatment failure, thus providing the possibility of changing the therapeutic scheme in a timely manner.

\section{MATERIALS \& METHODS}

\section{Clinical samples}

In this study we included samples from patients with parasitological diagnosis of CL caused by Leishmania panamensis as confirmed by PCR-RFLP, with a single lesion, an evolution period of less than 4 months, and a lesion area less than $4 \mathrm{~cm}^{2}$. The proteomic analysis was done with samples from 4 patients while the RT-qPCR analysis was carried out in samples from 5 patients.

\section{Sample preparation for proteomic analysis}

Granulation tissue from the center of the ulcer was collected before starting the treatment (TD0) and on day 7 of treatment (TD7). The material was placed in a vial containing $50 \mathrm{mM}$ Tris, 100 $\mathrm{mM} \mathrm{NaCl}, 20 \mathrm{mM}$ DTT, protease inhibitor and 1X phosphatases, with $\mathrm{pH} 7.5$ buffer. The samples were sonicated for 5 minutes at $4^{\circ} \mathrm{C}$ and subsequently centrifuged at 13,000 rpm for 20 $\min$ at $4{ }^{\circ} \mathrm{C}$; the supernatants were stored at $-80^{\circ} \mathrm{C}$ until use. The proteins were quantified, and their integrity was confirmed by SDS-PAGE.

\section{Immunoaffinity depletion}

Protein extracts were diluted 5-fold with 1X dilution buffer (Tris-Buffered Saline (TBS) $10 \mathrm{mM}$ Tris- $\mathrm{HCl}$ with $150 \mathrm{mM} \mathrm{NaCl}, \mathrm{pH}$ 7.4), filtered using a $0.45 \mu \mathrm{m}$ pore-size spin filter to remove particulate materials and centrifuged at $9,000 \times \mathrm{g}$ for $1 \mathrm{~min}$. Each of the diluted and filtered samples $(500 \mu \mathrm{l})$ were injected in a Seppro IgY 14 LC5 column with a constant flow rate of $0.5 \mathrm{ml} / \mathrm{min}$ for $20 \mathrm{~min}$, followed by a Seppro washing step at a flow rate of 2.0 $\mathrm{ml} / \mathrm{min}$ for $3 \mathrm{~min}$. Non-bound proteins (depleted fraction) were collected in the flow-through fraction. Due to the large volume of collected fractions, depleted samples were concentrated using Amicon ${ }^{\mathrm{TM}}$ Ultra-15 Centrifugal Filter Units (Millipore). Concentrated samples were reconstituted in a chaotropic buffer containing $8 \mathrm{M}$ urea, $2 \mathrm{M}$ thiourea, and $100 \mathrm{mM}$ 
122 triethylammonium bicarbonate $(\mathrm{TEAB})$ at $\mathrm{pH} 8.5$. Concentrated fractions were stored at $12380^{\circ} \mathrm{C}(18)$.

124

\section{In-gel protein digestion (Stacking gel)}

126 An aliquot concentrated flow-through fraction of each sample from four patients was diluted 127 with enough amount of sample loading buffer and then applied onto $1.2 \mathrm{~cm}$ wells of a 128 conventional SDS-PAGE gel ( $1 \mathrm{~mm}$ thick, $4 \%$ stacking, and $12 \%$ resolving). The run was 129 stopped when the sample front entered $1 \mathrm{~cm}$ into the resolving gel, to concentrate the whole 130 proteome in the stacking/resolving gel interface. The bands of unseparated protein were 131 visualized by Coomassie staining and then, bands were excised (19), cut into pieces ( $\left.1 \mathrm{~mm}^{2}\right)$, 132 deposited in 96-well plates, and processed automatically in a Proteineer DP (Bruker Daltonics, 133 Bremen, Germany). For digestion step, gel pieces were washed with $50 \mathrm{mM}$ ammonium 134 bicarbonate $\left(\mathrm{NH}_{4} \mathrm{HCO}_{3}\right)$ and then, with $\mathrm{ACN}$ prior to reduction with $10 \mathrm{mM}$ DTT in $25 \mathrm{mM}$ $135 \mathrm{NH}_{4} \mathrm{HCO}_{3}$. The alkylation was carried out with $55 \mathrm{mM}$ IAA in $50 \mathrm{mM} \mathrm{NH}_{4} \mathrm{HCO}_{3}$. Gel pieces 136 were then rinsed firstly with $50 \mathrm{mM} \mathrm{NH}_{4} \mathrm{HCO}_{3}$ and secondly with $\mathrm{ACN}$ and then were dried under a stream of nitrogen. Proteomics grade Trypsin (Sigma Aldrich) at a final concentration of $16 \mathrm{ng} / \mu \mathrm{l}$ in $25 \% \mathrm{ACN} / 50 \mathrm{mM} \mathrm{NH}_{4} \mathrm{HCO}_{3}$ was added and the digestion took place at $37^{\circ} \mathrm{C}$ for $4 \mathrm{~h}$.

139 The reaction was stopped by adding $50 \% \mathrm{ACN} / 0.5 \%$ TFA for peptide extraction. The tryptic eluted peptides were dried by speed-vacuum centrifugation and were cleaned up/desalted using

141 Stage-Tips with Empore 3M C18 disks (Sigma-Aldrich).

\section{Liquid chromatography and mass spectrometer analysis}

143 One $\mu \mathrm{g}$ aliquot of the previously digested sample from four patients was subjected to 1D-nano LC ESI-MS/MS analysis using a nano-liquid chromatography system (Eksigent Technologies nanoLC Ultra 1D plus, SCIEX, Foster City, CA) coupled to high-speed Triple TOF 5600 mass spectrometer (SCIEX, Foster City, CA) with a Nanospray III source. A silica-based reversed phase Acquity UPLC M-Class Peptide BEH C18 Column, $75 \mu \mathrm{m} \times 150 \mathrm{~mm}, 1.7 \mu \mathrm{m}$ particle size and $130 \AA$ pore size (Waters) was used as analytical column. The trapping column was a C18 Acclaim PepMap ${ }^{\mathrm{TM}} 100$ (Thermo Scientific), $100 \mu \mathrm{m} \times 2 \mathrm{~cm}, 5 \mu \mathrm{m}$ particle diameter, $100 \AA$ pore size. The loading pump delivered a solution of $0.1 \%$ formic acid in water at $2 \mu 1 / \mathrm{min}$. The nano-pump provided a flow-rate of $250 \mathrm{nl} / \mathrm{min}$ and was operated under gradient elution 
153 phase B (mobile phase A: 2\% acetonitrile, 0.1\% formic acid; mobile phase B: 100\% acetonitrile, $1540.1 \%$ formic acid). Injection volume was $5 \mu 1$.

155

156

157

158

159

160

161

162

163

164

165

166

167

168

169

170

171

172

173

174

175

176

177

178

179

180

181

182

183

A TripleTOF 5600 System (SCIEX, Foster City, CA) was used for data acquisition with an ion spray voltage floating (ISVF) $2300 \mathrm{~V}$, curtain gas (CUR) 35, interface heater temperature (IHT) 150, ion source gas 1 (GS1) 25, declustering potential (DP) $100 \mathrm{~V}$. All data were acquired using information-dependent acquisition (IDA) mode with Analyst TF 1.7 software (SCIEX, USA). For IDA parameters, $0.25 \mathrm{~s}$ MS survey scan in the mass range of 350-1250 Da were followed by $35 \mathrm{MS} / \mathrm{MS}$ scans of $100 \mathrm{~ms}$ in the mass range of 100-1800 (total cycle time: $4 \mathrm{~s}$ ). Switching criteria were set to ions greater than mass to charge ratio $(\mathrm{m} / \mathrm{z}) 350$ and smaller than $\mathrm{m} / \mathrm{z} 1250$ with the charge state of 2-5 and an abundance threshold of more than 90 counts (cps). Former target ions were excluded for $15 \mathrm{~s}$. IDA rolling collision energy (CE) parameters script was used for automatically controlling the $\mathrm{CE}(20)$.

\section{Quantification and data analysis}

The mass spectrometry data obtained were processed using PeakView ${ }^{\circledR} 2.2$ Software (SCIEX, Foster City, CA) and exported as mgf files. Proteomics data analysis were performed by using 4 search engines (Mascot Server v.2.6.1, OMSSA, X!Tandem, and MyriMatch) and a target/decoy database built from sequences in the Homo sapiens reference proteome at UniProt Knowledgebase. Search parameters included: trypsin as enzyme; 2 allowed missed cleavages; carbamidomethyl (C) as a fixed modification and acetyl (Protein N-term) and Oxidation (M) as variable modifications; peptide mass tolerance $\pm 25 \mathrm{ppm}$ for precursors and $0.02 \mathrm{Da}$ for fragment masses. Score distribution models were used to compute peptide-spectrum match p-values, and spectra recovered by a False Discovery Rate $(\mathrm{FDR})<=0.01$ (peptide-level) filter were selected for quantitative analysis (21).

\section{Label-free quantification}

Differential regulation was measured using linear models and the q-values (FDR) were used for determining statistical significance. All analyses were conducted using Proteobotics software (Madrid, Spain) (16). 
184 Pathway and interaction network analysis

185 The identified proteins were analyzed for biological function through the Panther Classification 186 System website (http://www.pantherdb.org). The proteins were categorized according to their 187 components, functions, and biological processes. The protein-protein interaction network (PPI) 188 was constructed using the online STRING database (https://string-db.org) (22).

189

190

\section{RNA isolation}

191 Five patients were included in the validation assay. The RNA was extracted using High Pure 192 RNA Tissue Kit (Roche, Mannheim, Germany), from scraping samples obtained on TD0 and 193 TD7. Samples were placed in $400 \mu 1$ of lysis/binding buffer and sonicated for 5 min in cold 194 room. Then, $200 \mu$ l ethanol was added and each sample was transferred to a High Pure filter 195 tube. After 30 min centrifugation at 13,000 x g, samples were treated with DNase for 15 min at $19625^{\circ} \mathrm{C}$. Five hundred $\mu \mathrm{l}$ wash buffer I were added and samples were centrifuged during $30 \mathrm{sec}$ at $1978,000 \times \mathrm{g}$. Washing step was repeated using $300 \mu \mathrm{l}$ of wash buffer II and centrifugation for 30 198 sec at 13,000 x g. Finally, the RNA was eluted with $30 \mu \mathrm{L}$ of elution buffer. A reverse 199 transcription reaction was performed in a $20 \mu 1$ volume in a PTC-100 Programmable Thermal 200 Controller (MJ Research, Inc., Quebec, Canada) using the Transcriptor First Strand cDNA 201 Synthesis kit (\#04379012001; Roche Diagnostics) according to the manufacturer's instructions, 202 with certain modifications.

203

204 cDNA synthesis

205 A reverse transcription reaction was performed in $20 \mathrm{ng}$ of RNA using the maximum first strand 206 cDNA synthesis kit, following the manufacturer's instructions: $4 \mu 1$ master mix reaction, $2 \mu 1$ RT 207 enzyme mix, $2 \mu 1$ RNA (DNase I treated), and $12 \mu$ water were mixed and incubated in the PTC $208100 \mathrm{TM}$ (MJ research) thermocycler for three cycles: $10 \mathrm{~min}$ at $25^{\circ} \mathrm{C}, 15 \mathrm{~min}$ at $50{ }^{\circ} \mathrm{C}$, and $5 \mathrm{~min}$ 209 at $85^{\circ} \mathrm{C}$, The obtained cDNA $(20 \mu \mathrm{l})$ was stored at $-20{ }^{\circ} \mathrm{C}$.

212 Each reaction mixture consisted of $2 \mu 1$ reverse transcription reaction product as the template, 0.5 $213 \mu 1$ each of forward and reverse primers, $7 \mu 1$ PCR grade water and $10 \mu 1$ of QuantiTect SYBR 214 Green PCR Kits (Qiagen, Hilden, Germany). The total volume of the reaction was $20 \mu 1$. The 
215 RT-qPCR reaction was completed with an initial denaturation step ( $95^{\circ} \mathrm{C}$ for $15 \mathrm{~min}$ ) followed 216 by 40 cycles of denaturation $\left(95^{\circ} \mathrm{C}, 10 \mathrm{sec}\right)$, annealing $\left(60^{\circ} \mathrm{C}, 30 \mathrm{sec}\right)$ and extension $\left(72{ }^{\circ} \mathrm{C}, 30\right.$ $217 \mathrm{sec}$ ). A final cooling step was conducted at $72{ }^{\circ} \mathrm{C}$ for $300 \mathrm{sec}$. The RT-qPCR reaction was 218 performed using the LightCycler 96 Instrument. Each sample was analyzed in duplicate.

219

220

221

222

223

224

225

226

227

228

229

230

231

232

233

234

235

236

237

238

239

\section{0}

241

242

243

244

245

Three genes from up regulated proteins SERPINB1, S100A8, and S100A9 were selected for the measurement of relative expression. The Glyceraldehyde-3-phosphate dehydrogenase (GAPDH) gene was used as constitutive gene. The primers were designed using Primer 3 software (bioinfo.ut.ee/primer3-0.4.0/). The following primer sequences were used: SERPINB1, Fwd 5'ATGAAAGAAGCCACGACGAA-3' and Rev 5'- GGTAAGGCAGTTCCAGCACA-3'; S100A8, Fwd 5'- GCCAAGCCTAACCGCTAT-3' and Rev 5'CCCACCTGAAAAACAGAACC-3'; S100A9, Fwd 5'- GCACCCCCTAAGACCACAG-3' and Rev 5'- CCCCACAGCCAAGACAGTT-3'; and GAPDH, Fwd 5'GCTTCGCTCTCTGCTCCTC-3' and Rev 5'- ACGACCAAATCCGTTGACTC-3'.

\section{Ethics Statement}

All study subjects provided their informed written consent to be included in the study. The study was approved by the Human Bioethics Committee of Universidad de Antioquia - SIU (Act 007-2017).

\section{RESULTS}

All patients were treated with meglumine antimoniate. The cure was monitored for six months (until PTD 180). By this time all patients healed their lesion. No reactivation was detected (Figure 1).

\section{Proteins detected in granulation tissue of CL}

In total, 344 proteins were identified in the granulation tissue formed in the center of the ulcer. After analyzing the quality of the data obtained in MS, three of the four biological replicas were selected for label-free quantification by Q-values (FDR) using Proteobotics software (Madrid, Spain). The label-free quantification analyses yielded 267 proteins that were truly quantified in the three biological samples $(\mathrm{p}<0.05)$. Of the 267 proteins, 86 proteins were up-regulated 
$246(32.21 \%$ ratio $>0.67), 59$ proteins were down-regulated $(22.09 \%$ ratio $<0.717)$ and 122 proteins 247 showed no changes (44.9\%). The 267 proteins were grouped according to their cellular locations 248 as follows: 63 intracellular, 4 intracellular membranes, 53 extracellular membranes, 6 249 extracellular matrices, 56 extracellular space, 43 macromolecular complex and 42 organelles 250 (Figure 2A). The proteins were also classified according to their Class: 21 Calcium binding 251 protein, 11 Chaperone, 13 Cytoskeletal protein, 14 Defense/immunity protein, 33 Enzyme 252 modulator, 18 Hydrolase, 10 Membrane traffic protein, 13 Nucleic acid binding, 22 Receiver, 253112 Other (Figure 2B). Finally, they were also classified according to their biological process: 254 Antioxidant activity 7, Binding protein 89, Catalytic activity 59, Receptor activity 2, Structural 255 molecule activity 13, Translation regulatory activity 1, Transporter activity 5 and Other 91 256 (Figure 2C).

257

\section{Label-free quantification}

259 Using the FDR analysis, 12 proteins were found with differential expression when comparing 260 TD0 and TD7. Five were down-regulated (FABP5, S100A2, PRDX2, HBA1, and HBD) and 7 261 were up-regulated (KRT73, LAMP2, S100A9, S100A8, KRT10, SERPINB1, and ATP7A). All

262 differentially expressed proteins were classified using the Panther Classification System website 263 (http://www.pantherdb.org).

264

The classification according to their molecular function showed a distribution between fatty acid binding 3, Toll-like receptor 4 binding 2, arachidonic acid binding 2, RAGE receptor binding 2, and oxygen transporter activity 2 (Figure 3 ). The classification of the cellular components showed that all proteins are located at the extracellular level: extracellular region 10 genes, extracellular exosome 9 genes, extracellular region 10 genes, extracellular space 6 genes and membrane-bound vesicle 8 genes.

271

272 The classification according to their biological process showed that these proteins participate in 273 neutrophil aggregation and degranulation, sequestering of zinc, peptidyl-cysteine S-nitrosylation, 274 chemokine production, leukocyte migration during the inflammatory response, removal of 275 superoxide radicals, oxygen transport, hydrogen peroxide catabolic process, astrocyte 276 development, antimicrobial humoral response and epidermis development. The proteins showed 
277 a fold enrichment between 17.1 and $100 \%$, the p-log 10 value between 3.9 and 5.7 and an FDR $278<0.05$ (Table 1).

279

280

281

\section{Interaction network analysis}

282

The proteins were analyzed to determine the functional interaction network that unites the 12

283

284

285

286

287

288

289

290

291

292

293

294

295

296

297

298

299

300

301

302

303

304

305

306

307

proteins differentially expressed between TD0 and TD7 using STRING database, obtaining a minimum required interaction score of 0.4 (medium confidence). The analysis showed that 5 of the 12 proteins had a good correlation and were classified in 2 groups. In the first group were S100A9, S100A8, and S100A2, all calcium-binding proteins. In the second group were HBD and HBA2, both components of hemoglobin (alpha and delta) (Figure 4).

\section{Relative expression by RT-qPCR}

The levels of expression of SERPINB1, S100A8, S100A9 at TD7 were compared with respect to the levels before treatment (TD0) using the $\Delta \Delta \mathrm{CT}$ method. All 5 patients were cured after 6 months of follow-up. The expression levels increased $2.65 \pm 1.22$ times for SERPINB1, $3.2 \pm$ 2.81 for S100A8, $1.38 \pm 0.67$ for S100A9 (Figure 5).

\section{DISCUSSION}

Leishmaniasis is one of the seven main tropical diseases and continues to be one of the major public health problems around the world (23). Reevaluation of treatment guidelines for cutaneous leishmaniasis is urgently needed because there is high risk of toxicity of pentavalent antimony and treatment failure is increasing $(24,25)$.

The major adverse effects associated with pentavalent antimonial treatments are local pain, arthralgia, myalgia, increases in hepatic enzymes, urea, and creatinine, and electrocardiographic alterations (inversion of the T wave, prolongation of the Q-T segment, depression of the S-T segment, and sinus bradycardia). Based on their severity, it is necessary to stop the treatment and modify the treatment scheme or change the drug (26).

The search for biomarkers in leishmaniasis has emerged as a need to not only identify markers of susceptibility and resistance to infection or the evaluation of vaccines effectiveness, but also to 
308 monitor early and late therapeutic efficacy $(5-7,9)$. However, none of the proposed biomarkers

309 has been validated.

310

311 For monitoring of the therapeutic response of cutaneous leishmaniasis, various biomarkers were

312 proposed. . These biomarkers include the parasite load, levels of IL4, IL8, INF $\gamma$, MMP2

313 expression in skin biopsies and also levels of TNF $\alpha$, IL10, IL15, IL32 $\gamma$, TGF $\beta$, sCD26, sCD30,

314 cortisol, prolactin and SOD1 in serum (27). Nevertheless, the applicability of these proposed

315 biomarkers is still debatable either because of the difficulty of collecting samples from invasive

316 biopsies, or because the cytokines detected in serum cannot be attributed to a specific infectious

317 process located on the skin as would be in the case of CL, where it can be influenced by

318 conditions particular to each individual.

319

320 Little has been explored in the search for early biomarkers of therapeutic response to predict the

321 effectiveness of treatment in the first week. The biomarkers mentioned above were detected in

322 skin biopsies and serum samples before treatment and their previous levels correlated with the

323 therapeutic response at the end of treatment. In our approach, we use a sample obtained of the

324 center of the ulcer by scraping that does not generate pain or discomfort in the patient.

325 Additionally, the material is obtained from the site where both the damage and repair process

326 occur, during a curative therapeutic response.

327

328 The healing proteome has been studied in different animal models, especially in mice and pigs.

329 These studies showed that proteins with differential expression correspond to receptors,

330 hydrolases, enzymes, immunity, cytoskeletal proteins, transporters and oxidoreductases $(28,29)$.

331 Additionally, in pathogenic processes such as type 2 diabetes and chronic ulcers, where there is

332 an increase in expression levels of proteins related to inflammation and tissue destruction,

333 proteins with differential expression have been identified, most of which relate to angiogenesis,

334 cell death and inflammation $(30,31)$.

335

336 In CL, where scarring of the lesion is distorted by the presence of the parasite and the

337 inflammatory phase is perpetuated over time, the healing process only begins with the

338 disappearance of the antigenic stimulus triggered by the parasite. 
340 Our results showed differential expression in 12 proteins when comparing expression levels on

341 TD0 and TD7. Five were down-regulated and seven were up-regulated. The up-regulated

342 proteins participate in biological processes such as neutrophil aggregation, response to zinc ion,

343 sequestering of zinc ion, peptidyl-cysteine S-nitrosylation and cellular transition metal ion

344 homeostasis, the down-regulated proteins participate in oxygen transport and hydrogen peroxide 345 catabolism.

346

347 There was a decrease in the expression of FABP5 and S100A2. FABP5 is a fatty acid binding 348 protein while S100A2 is a calcium transport protein. Both proteins show increased levels in 349 psoriasis (32), revealing their role in the inflammatory process (33). Thus, the decrease in 350 FABP5 and S100A2 in the case of CL would correlate with a regulation of the inflammatory 351 stage. On the other hand, PRDX2 is a peroxidase which has been considered a biomarker in 352 inflammatory processes such as multiple sclerosis (34). Its early decline in CL also confirms a 353 regulation of the inflammatory process. Finally, HBA1 and HBD are subunits of hemoglobin, 354 which is highly important in oxygen transport that favors oxidative response. Decrease of HBA1 355 and HBD levels in tissue can be correlated with a reduction of the oxidative response and 356 therefore of the damage generated in the tissue by oxidative mechanisms. Else, the low levels of 357 these proteins in skin could be related to their inability to regulate oxidative response similar to 358 that observed in Steatohepatitis (35).

359

360 Change in the protein profiles with increased Keratin, type II cytoskeletal 73, a protein present in 361 the hair follicle (related to keratinization and important in the development of the skin in mice 362 (36), confirms that this protein would be participating early in the new tissue formation. On the 363 other hand, LAMP2 is a glycoprotein that has been described as important in processes mediated 364 by lysosomes. In the context of healing, it has been described in squamous cell carcinoma where 365 its expression is related to cell adhesion and cell migration, generating a layer of glycocalyx that 366 prevents the degradation of cells and the action of different proteases (37). Thus, in the case of

367 CL it may be participating in the process of tissue repair, possibly in cell migration. 
369 S100A9 and S100A8 are calcium- and zinc-binding proteins related to different processes such

370 as the regulation of inflammation, cell cycle progression and cell differentiation, induction of

371 neutrophil chemotaxis, phagocytosis, as well as degranulation (38). Additionally, they are

372 overexpressed during the healing process (30), particularly in the differentiated keratinocytes and

373 their participation in the reorganization of the keratin cytoskeleton in the injured epidermis is

374 well-established (39). Based on their roles in healing, we believe that their increase in early

375 response to treatment correlates with the beginning of the process of tissue repair.

376

377 KRT10 is a type of keratin that has been described as an early marker of daughter cell

378 differentiation in the basal stratum that faces the epidermal surface and contributes to re-

379 epithelialization. Studies in mice confirmed that the absence of cells expressing KRT10

380 correlates with deterioration in re-epithelialization (40). Therefore, in CL, the increase in the

381 expression of KRT10 would be correlated with the beginning of the re-epithelialization process.

382 SERPINB1 belongs to the superfamily of serine protease inhibitors, a protein that has various

383 functions. In the inflammatory process it has been shown to play an important role inhibiting

384 apoptosis, activation of caspase 3 and the action of different proteases such as elastase, cathepsin

$385 \mathrm{G}$ and D, and proteinase-3 (41). It has been demonstrated in models of corneal epithelium in

386 cattle, that during cicatrization SERPINB1 participates in cell migration and its increased level is

387 related to the closure of the wound; the levels are restored to normal values when the injury has

388 healed (42). Both mechanisms would be important in the therapeutic response in CL since

389 increased apoptosis has been described as one of the pathogenesis markers in proteome studies of

390 pathogenesis in CL (43). On the other hand, cell migration and wound closure would be related

391 to the start of the lesion healing.

392

393 Finally, ATP7A is a very interesting protein for the regulation of intracellular copper

394 concentrations, fulfilling two very important functions: the elimination of the accumulated

395 intracellular copper and the transportation of the copper necessary for the proper functioning of

396 the copper-dependent enzymes (44). The human peptide Gly- (L-His) - (L-Lys) or GHK has a

397 high affinity for copper as a carrier and once it binds copper, it may have an anti-inflammatory

398 effect with suppression of free radicals, formation of thromboxane, release of iron oxidant and

399 TGF $\beta$, increase of superoxide dismutase and vasodilation, which would be essential for tissue 
400 repair in LC. It also has a chemoattractant effect on macrophages, mast cells and capillary cells

401 (45). All these effects are essential for the entire process of tissue repair in CL.

402 The wound healing process includes homeostasis, inflammation, proliferation and remodeling. In 403 the case of CL, once the infection is controlled by the treatment, the inflammatory phase ends 404 and, the oxidative response and the production of proinflammatory molecules involved in tissue 405 damage disappear. The oxidative response is regulated by a decrease of HBA1, HBD and 406 PRDX2. While HBA1 and HBD are essential for oxygen transportation, PRDX2 is essential for 407 the oxidative response. The relationship between HBA1 and HBD with PRDX2 is also 408 demonstrated in chronic inflammatory processes such as Bowel's disease (46). On the other 409 hand, FABP5 can regulate the production of prostaglandin E2 (47) and S100A2 is associated 410 with inflammatory processes such as periodontitis and constitutes a candidate for biomarker of 411 inflammation in this context (48). The repair process begins with migration, adhesion, 412 proliferation and cell differentiation. The GHK controls the migration of macrophages and the 413 macrophages differentiate to M2 type to increase the production of TGF $\beta$ (45). SERPINB1 414 attract fibroblasts to the wound healing site (42) and LAMP2 promotes the extracellular matrix 415 (37). Lastly, KRT10, S100A9 and S100A8 induce proliferation and differentiation of fibroblast $416(30,40)$ and then, occur the re-epithelialization with the participation of keratin and type II 417 cytoskeletal 73 protein.

418 The identification of these proteins with increased expression after healing are closely related to 419 the beginning of the cell proliferation stage when the infection and inflammation is controlled. 420 Although unexpected, we do not find proteins belonging to the parasite in the ulcer center, 421 possibly because the proportion of infected cells in the ulcer is lower than in the active borders. 422 The measurement by RT-qPCR showed an induction level of S100A8 $>2$ in the cured patients 423 suggesting that SERPINB1 and S100A8 are good candidates for an early biomarker of healing. 424 In turn, the induction level of S100A9 was $<2$, indicating that this marker is not a good 425 candidate. Nevertheless, before accepting or ruling out any of these candidates for biomarkers, 426 these results need to be validated in a larger group of patients.

\section{CONCLUSION}

429 In conclusion, our work allowed for two very important analyses. The first is the characterization 430 of proteome of pathogenesis in the ulcer generated in CL and the second is the identification of 
431 the changes in the protein profile in a positive therapeutic response to treatment, allowing to

432 identify pathways related to inflammation and a negatively regulated oxidative response, usually 433 associated with the reduction of inflammation and increased tissue repair. With these results, it is 434 possible to propose a multicentric study that allows the validation of the healing biomarkers by 435 RT-qPCR for the early prediction of the treatment. These results will allow clinicians to 436 implement, in a timely manner, an appropriate strategy to guarantee a successful treatment.

437

438 Acknowledgements

439 Authors thanks Juliana Quintero, MD for the assistance during patients care.

440

441 Financial support

442 This work and A.M. received financial support from El Departamento Administrativo de 443 Ciencia, Tecnología e Innovación -Colciencias (CT-695-2014)

444

445 Conflict of interest

446 We have no conflicts of interest to declare.

447

448

References

449

450

451

452

453

454

455

456

457

458

1. Bates P a. 2007. Transmission of Leishmania metacyclic promastigotes by phlebotomine sand flies. International Journal for Parasitology 37:1097-1106. DOI: 10.1016/j.ijpara.2007.04.003.

2. Alvar J., Vélez ID., Bern C., Herrero M., Desjeux P., Cano J., Jannin J., Boer M den. 2012. Leishmaniasis Worldwide and Global Estimates of Its Incidence. PLoS ONE 7:e35671. DOI: 10.1371/journal.pone.0035671.

3. Handler MZ., Patel P a., Kapila R., Al-Qubati Y., Schwartz R a. 2015. Cutaneous and mucocutaneous leishmaniasis. Journal of the American Academy of Dermatology 73:911-926. DOI: 10.1016/j.jaad.2014.09.014.

459

4. Oliaee RT., Sharifi I., Afgar A., Kareshk AT., Asadi A., Heshmatkhah A., Bamorovat M., Jafarzadeh A., Mohammadi MA., Daneshvar H. 2018. Unresponsiveness to meglumine antimoniate in anthroponotic cutaneous leishmaniasis field isolates: analysis 
461

462

463

464

465

466

467

468

469

470

471

472

473

474

475

476

477

478

479

480

481

482

483

484

485

486

487

488

489

490

491

of resistance biomarkers by gene expression profiling. Tropical Medicine \& International Health. DOI: 10.1111/tmi.13062.

5. Portela ÁSB., Costa LE., Salles BCS., Lima MP., Santos TTO., Ramos FF., Lage DP., Martins VT., Caligiorne RB., Lessa DR., Silva FR., Machado AS., Nascimento GF., Gama IS., Chávez-Fumagalli MA., Teixeira AL., Rocha MOC., Rocha RL., Coelho EAF. 2018. Identification of immune biomarkers related to disease progression and treatment efficacy in human visceral leishmaniasis. Immunobiology 223:303-309. DOI: 10.1016/j.imbio.2017.10.043.

6. Martinez-Subiela S., Horvatic A., Escribano D., Pardo-Marin L., Kocaturk M., Mrljak V., Burchmore R., Ceron JJ., Yilmaz Z. 2017. Identification of novel biomarkers for treatment monitoring in canine leishmaniosis by high-resolution quantitative proteomic analysis. Veterinary Immunology and Immunopathology 191:60-67. DOI: 10.1016/j.vetimm.2017.08.004.

7. Corpas-lópez V., Merino-espinosa G., Acedo-sánchez C., Díaz-sáez V. 2016. Veterinary Parasitology Hair parasite load as a new biomarker for monitoring treatment response in canine leishmaniasis. Veterinary Parasitology 223:20-25. DOI: 10.1016/j.vetpar.2016.04.001.

8. Ibarra-Meneses A V., Ghosh P., Hossain F., Chowdhury R., Mondal D., Alvar J., Moreno J., Carrillo E. 2017. IFN- $\gamma$, IL-2, IP-10, and MIG as Biomarkers of Exposure to Leishmania spp., and of Cure in Human Visceral Leishmaniasis. Frontiers in Cellular and Infection Microbiology 7:1-8. DOI: 10.3389/fcimb.2017.00200.

9. Kammoun-rebai W., Naouar I., Libri V., Albert M., Louzir H. 2016. Protein biomarkers discriminate Leishmania major -infected and non-infected individuals in areas endemic for cutaneous leishmaniasis. BMC Infectious Diseases:1-10. DOI: 10.1186/s12879-0161458-6.

10. Kip AE., Balasegaram M., Beijnen JH., Schellens JHM., de Vries PJ., Dorlo TPC. 2015. Systematic Review of Biomarkers To Monitor Therapeutic Response in Leishmaniasis. Antimicrobial Agents and Chemotherapy 59:1-14. DOI: 10.1128/AAC.04298-14.

11. Carvalho EM., Valenzuela JG., Oliveira CI De. 2017. Seroconversion to Lutzomyia intermedia LinB-13 as a biomarker for developing cutaneous leishmaniasis. Scientific Reports 1-10. DOI: 10.1038/s41598-017-03345-0. 
492

493

494

495

496

497

498

499

500

501

502

503

504

505

506

507

508

509

510

511

512

513

514

515

516

517

518

519

520

521

522

523

524

525

12. Ong S., Mann M. 2005. Mass spectrometry - based proteomics turns quantitative. 1:252262. DOI: $10.1038 /$ nchembio736.

13. Bantscheff M., Lemeer S. 2012. Quantitative mass spectrometry in proteomics : critical review update from 2007 to the present. :939-965. DOI: 10.1007/s00216-012-6203-4.

14. Pottiez G., Wiederin J., Fox HS., Ciborowski P. 2012. Comparison of 4-plex to 8-plex iTRAQ Quantitative Measurements of Proteins in Human Plasma Samples. J Proteome Res: 6;11(7):3774-81. doi: 10.1021/pr300414z.

15. Thompson A., Kuhn K., Kienle S., Schwarz J., Neumann T., Hamon C. 2003. Tandem Mass Tags : A Novel Quantification Strategy for Comparative Analysis of Complex Protein Mixtures by MS / MS. Analytical Chemistry. 75:1895-1904. DOI: 10.1021/ac0262560.

16. Griffin NM., Yu J., Long F., Oh P., Shore S., Li Y., Koziol JA., Schnitzer JE. 2010. Label-free, normalized quantification of complex mass spectrometry data for proteomic analysis. Nature Biotechnology 28:83-89. DOI: 10.1038/nbt.1592.

17. Atrih A., Mudaliar MA V., Zakikhani P., Lamont DJ., Huang JTJ., Bray SE., Barton G., Fleming S., Nabi G. 2014. Quantitative proteomics in resected renal cancer tissue for biomarker discovery and profiling. British Journal of Cancer 110:1622-1633. DOI: 10.1038/bjc.2014.24.

18. Hyung SW, Piehowski PD, Moore RJ, Orton DJ, Schepmoes AA, Clauss TR, Chu RK,Fillmore TL, Brewer H, Liu T, Zhao R, Smith RD. 2014. Microscale depletion of high abundance proteins in human biofluids using IgY14 immunoaffinity resin: analysis of human plasma and cerebrospinal fluid. Anal Bioanal Chem. 406(28):7117-25. DOI: 10.1007/s00216-014-8058-3.

19. Andreu Z, Otta Oshiro R, Redruello A, López-Martín S, Gutiérrez-Vázquez C, Morato E, Marina AI, Olivier Gómez C, Yáñez-Mó M. 2017. Extracellular vesicles as a source for non-invasive biomarkers in bladder cancer progression. Eur J Pharm. 15;98:70-79. DOI: 10.1016/j.ejps.2016.10.008.

20. Segura V, Medina-Aunon JA, Guruceaga E, Gharbi SI, González-Tejedo C, Sánchez del Pino MM, Canals F, Fuentes M, Casal JI, Martínez-Bartolomé S, Elortza F, Mato JM, Arizmendi JM, Abian J, Oliveira E, Gil C, Vivanco F, Blanco F, Albar JP, Corrales FJ. 2013. Spanish human proteome project: dissection of chromosome 16. J Proteome Res. 12(1):112-22. DOI: 10.1021/pr300898u.

21. Li S, Cao Q, Xiao W, Guo Y, Yang Y, Duan X, Shui W. 2017. Optimization of Acquisition and Data-Processing Parameters for Improved Proteomic Quantification by 
526

527

528

529

530

531

532

533

534

535

536

537

538

539

540

541

542

543

544

545

546

547

548

549

550

551

552

553

554

555

556

557

Sequential Window Acquisition of All Theoretical Fragment Ion Mass Spectrometry. $J$ Proteome Res. 16(2):738-747. DOI: 10.1021/acs.jproteome.6b00767.

22. Wu X, Hasan MA, Chen JY. 2014. Pathway and network analysis in proteomics. J Theor Biol. 7;362:44-52. doi: 10.1016/j.jtbi.2014.05.031.

23. Torres-Guerrero E., Quintanilla-Cedillo MR., Ruiz-Esmenjaud J., Arenas R. 2017. Leishmaniasis: a review. F1000Research 6:750. DOI: 10.12688/f1000research.11120.1. 24. Von Stebut E. 2015. Leishmaniasis. JDDG: Journal der Deutschen Dermatologischen Gesellschaft 13:191-201. DOI: 10.1111/ddg.12595.

25. López-Carvajal L. 2015. Systematization of Clinical Studies on the Efficacy of Treatments for Cutaneous Leishmaniasis 1980-2015 Resumen. Acta Reumatológica:111. DOI: $10.3823 / 1307$.

26. Romero GAS., Costa DL., Costa CHN., de Almeida RP., de Melo EV., de Carvalho SFG., Rabello A., de Carvalho AL., Sousa A de Q., Leite RD., Lima SS., Amaral TA., Alves FP., Rode J. 2017. Efficacy and safety of available treatments for visceral leishmaniasis in Brazil: A multicenter, randomized, open label trial. PLoS Neglected Tropical Diseases 11:1-25. DOI: 10.1371/journal.pntd.0005706.

27. Kip AE., Balasegaram M., Beijnen JH., Schellens JHM., de Vries PJ., Dorlo TPC. 2015. Systematic Review of Biomarkers To Monitor Therapeutic Response in Leishmaniasis. Antimicrobial Agents and Chemotherapy 59:1-14. DOI: 10.1128/AAC.04298-14.

28. Chen L., Hou Q., Zhou Z-Z., Li M-R., Zhong L-Z., Deng X-D., Zhu Z-Y., Cheng Z-Y., Zhu J., Xiang C-L., He W-J., Fu X-B. 2017. Comparative Proteomic Analysis of the Effect of the Four-Herb Chinese Medicine ANBP on Promoting Mouse Skin Wound Healing. The international journal of lower extremity wounds 16:154-162. DOI: 10.1177/1534734617720623.

29. Sabino F., Hermes O., Egli FE., Kockmann T., Schlage P., Croizat P., Kizhakkedathu JN., Smola H., auf dem Keller U. 2015. In Vivo Assessment of Protease Dynamics in Cutaneous Wound Healing by Degradomics Analysis of Porcine Wound Exudates. Molecular \& Cellular Proteomics 14:354-370. DOI: 10.1074/mcp.M114.043414.

30. Eming SA., Koch M., Krieger A., Brachvogel B., Kreft S., Bruckner-Tuderman L., Krieg T., Shannon JD., Fox JW. 2010. Differential proteomic analysis distinguishes tissue repair biomarker signatures in wound exudates obtained from normal healing and chronic wounds. Journal of Proteome Research 9:4758-4766. DOI: 10.1021/pr100456d. 
558

559

560

561

562

563

564

565

566

567

568

569

570

571

572

573

574

575

576

577

578

579

580

581

582

583

584

585

586

587

588

589

31. Krisp C., Jacobsen F., Mckay MJ., Molloy MP., Steinstraesser L., Wolters DA. 2013. Proteome analysis reveals antiangiogenic environments in chronic wounds of diabetes mellitus type 2 patients. Proteomics 13:2670-2681. DOI: 10.1002/pmic.201200502.

32. Chamcheu J., Chaves-Rodriquez M-I., Adhami V., Siddiqui I., Wood G., Longley B., Mukhtar H. 2014. Upregulation of PI3K/AKT/mTOR, FABP5 and PPAR $\beta / \delta$ in Human Psoriasis and Imiquimod-induced Murine Psoriasiform Dermatitis Model. Acta Dermato Venereologica 96:854-6. DOI: 10.2340/00015555-2359.

33. Eckert RL., Broome A-M., Ruse M., Robinson N., Ryan D., Lee K. 2004. S100 Proteins in the Epidermis. Journal of Investigative Dermatology 123:23-33. DOI: 10.1111/j.0022202X.2004.22719.x.

34. Voigt D., Scheidt U., Derfuss T., Brück W., Junker A. 2017. Expression of the Antioxidative Enzyme Peroxiredoxin 2 in Multiple Sclerosis Lesions in Relation to Inflammation. International Journal of Molecular Sciences 18:760. DOI: 10.3390/ijms 18040760 .

35. Liu W., Baker SS., Baker RD., Nowak NJ., Zhu L. 2011. Upregulation of Hemoglobin Expression by Oxidative Stress in Hepatocytes and Its Implication in Nonalcoholic Steatohepatitis. PLoS ONE 6:e24363. DOI: 10.1371/journal.pone.0024363.

36. Liu B., Xu Y., Li W., Zeng L. 2015. Proteomic analysis of differentially expressed skin proteins in iRhom2(Uncv) mice. BMB reports 48:19-24. DOI: 10.5483/BMBRep.2015.48.1.022

37. Li L., Wang W., Zhang R., Liu J., Yu J., Wu X., Xu Y., Ma M., Huang J. 2017. High expression of LAMP2 predicts poor prognosis in patients with esophageal squamous cell carcinoma. Cancer Biomarkers 19:305-311. DOI: 10.3233/CBM-160469.

38. Zhong A., Xu W., Zhao J., Xie P., Jia S., Sun J., Galiano RD., Mustoe TA., Hong SJ. 2016. S100A8 and S100A9 Are Induced by Decreased Hydration in the Epidermis and Promote Fibroblast Activation and Fibrosis in the Dermis. American Journal of Pathology 186:109-122. DOI: 10.1016/j.ajpath.2015.09.005.

39. Thorey IS., Roth J., Regenbogen J., Halle J-P., Bittner M., Vogl T., Kaesler S., Bugnon P., Reitmaier B., Durka S., Graf A., Wöckner M., Rieger N., Konstantinow A., Wolf E., Goppelt A., Werner S. 2001. The Ca 2+ -binding Proteins S100A8 and S100A9 Are Encoded by Novel Injury-regulated Genes. Journal of Biological Chemistry 276:3581835825. DOI: 10.1074/jbc.M104871200. 
590

591

592

593

594

595

596

597

598

599

600

601

602

603

604

605

606

607

608

609

610

611

612

613

614

615

616

617

618

619

40. Zhao H., Rieger S., Abe K., Hewison M., Lisse TS. 2016. DNA damage-inducible transcript 4 is an innate surveillant of hair follicular stress in vitamin D receptor knockout mice and a regulator of wound re-epithelialization.hair International Journal of Molecular Sciences 17. DOI: 10.3390/ijms17121984.

41. Loison F., Xu Y., Luo HR., Diseases B. 2014. Proteinase 3 and Serpin B1: a novel pathway in the regulation of caspase-3 activation, neutrophil spontaneous apoptosis, and inflammation. Inflammation and Cell Signaling 1:1-5. DOI: 10.14800/ics.462.

42. Torriglia A., Martin E., Jaadane I. 2017. The hidden side of SERPINB1/Leukocyte Elastase Inhibitor. Seminars in Cell and Developmental Biology 62:178-186. DOI: 10.1016/j.semcdb.2016.07.010.

43. Da C., Santos S., Attarha S., Saini RK., Boaventura V., Costa J., Khouri R., Barral-Netto M., Brodskyn CI., Souchelnytskyi S. 2015. Proteome Profiling of Human Cutaneous Leishmaniasis Lesion. Journal of Investigative Dermatology 135:400-410. DOI: 10.1038/jid.2014.396.

44. Da C., Santos S., Attarha S., Saini RK., Boaventura V., Costa J., Khouri R., Barral-Netto M., Brodskyn CI., Souchelnytskyi S. 2015. Proteome Profiling of Human Cutaneous Leishmaniasis Lesion. Journal of Investigative Dermatology 135:400-410. DOI: 10.1038/jid.2014.396.

45. Da C., Santos S., Attarha S., Saini RK., Boaventura V., Costa J., Khouri R., Barral-Netto M., Brodskyn CI., Souchelnytskyi S. 2015. Proteome Profiling of Human Cutaneous Leishmaniasis Lesion. Journal of Investigative Dermatology 135:400-410. DOI: 10.1038/jid.2014.396.

46. Senhaji N, Zaid Y, El Khalfi B, Fahimi M, Martin J, Badre W, Nadifi S, Soukri A. 2017. Peroxiredoxin-2 up-regulation in inflammatory bowel disease: Friend or foe? Journal of Gastroenterology and Hepatology. 32(6):1212-1220. DOI: 10.1111/jgh.13664.

47. Bogdan D, Falcone J, Kanjiya MP, Park SH, Carbonetti G, Studholme K, Gomez M, Lu Y, Elmes MW, Smietalo N, Yan S, Ojima I, Puopolo M, Kaczocha M. 2018. Fatty acidbinding protein 5 controls microsomal prostaglandin E synthase 1 (mPGES-1) induction during inflammation. Journal of Biological Chemistry. 293(14):5295-5306.

DOI:10.1074/jbc.RA118.001593. 
620

621

622
48. Heo SH, Choi YJ, Lee JH, Lee JM, Cho JY. 2011. S100A2 level changes are related to human periodontitis. Molecules and Cells. 32(5):445-50. doi: 10.1007/s10059-011-01325. 


\section{Table $\mathbf{1}$ (on next page)}

Table 1. Classification of proteins with differential expression according to the complete biological process*.

* Panther classification system 
1 Table 1. Classification of proteins with differential expression according to the 2 complete biological process*.

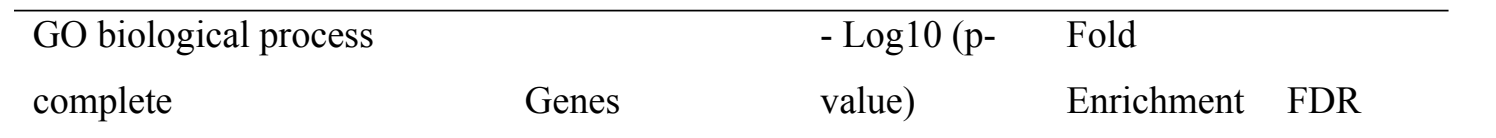

Neutrophil aggregation

(GO:0070488)

P06702, P05109 5,7

$>100$

0,00464

Sequestering of zinc ion

(GO:0032119)

P06702,P05109 5,4

$>100$

0,00696

Peptidyl-cysteine S-

nitrosylation (GO:0018119)

P06702,P05109 5,

0,00683

Chemokine production

(GO:0032602)

P06702,P05109

4,7

$>100$

0,0122

Leukocyte migration

involved in inflammatory

response (GO:0002523)

P06702,P05109

4,6

$>100$

0,0134

Removal of superoxide

radicals (GO:0019430)

P32119,Q04656

4,5

$>100$

0,0152

Oxygen transport

(GO:0015671)

P69905,P02042

4,4

$>100$

0,0163

Hydrogen peroxide catabolic process (GO:0042744)

P32119,P69905

4,2

$>100$

0,0242

Astrocyte development

(GO:0014002)

P06702,P05109

3,9

$>100$

0,0426

Antimicrobial humoral

P06702,P05109,

response (GO:0019730)

Q04656

4,6

52,08

0,0134

Q01469,P13473

Neutrophil degranulation

,P06702,P05109

(GO:0043312)

,P30740

5,3

18,15

0,00643

Q04656,P13645

Epidermis development

,Q01469,Q86Y4

(GO:0008544)

6

4,2

17,11

0,0233

3

$4 *$ Panther classification system

5 


\section{Figure 1}

Evolution of ulcer in patients.

The figure shows the appearance of the cutaneous leishmanaisis during follow-up. TD0: before the start of treatment; TD7: on day 7 of treatment; PTD180: 6 months after the end of treatment.

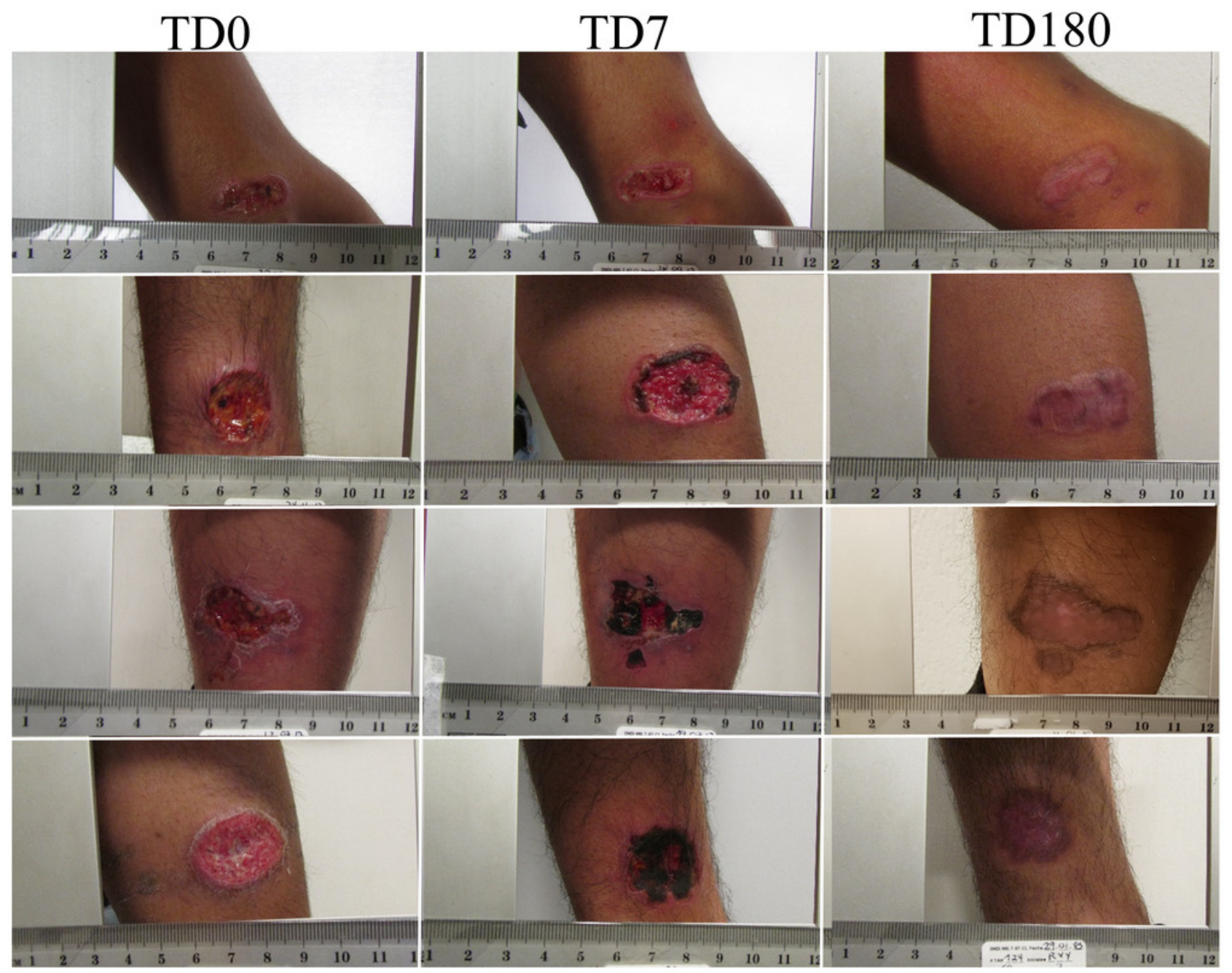


Figure 2

Global proteomic analysis of the granulation tissue.

Samples were obtained from a cutaneous leishmaniasis ulcer before treatment (TD0) and day

7 of treatment (TD7). The figure shows the gene ontology analysis of all the quantified

proteins. (A) Proteins present in cellular components. (B) protein classified in classes. (C) Molecular function of proteins.. 
A

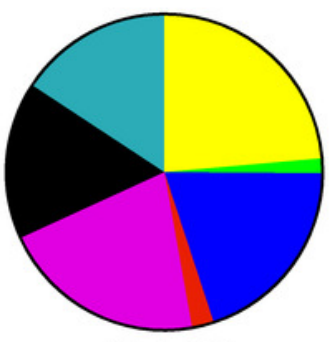

$$
\text { Total }=267
$$

$23.60 \% 63$ Intracellular

$\square 1.50 \% 4$ Intracellular membrane

- $19.85 \% 53$ Extracellular membrane

$\square 2.25 \% 6$ Extracellular matrix

$\square 20.97 \% 56$ Extracellular space

- $16.10 \% 43$ Macromolecular complex

$\square 15.73 \% 42$ Organelle

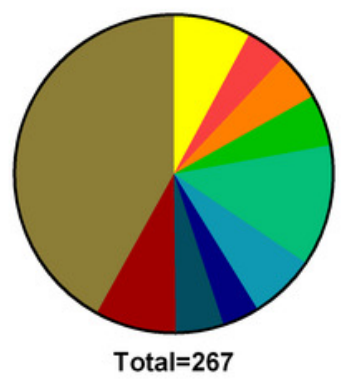

$\square 7.87 \% 21$ Calcium binding protein $\square 4.12 \% 11$ Chaperone

$\square 4.87 \% 13$ Cytoskeletal protein

$\square 5.24 \% 14$ Defense/immunity protein

$\square 12.36 \% 33$ Enzyme modulator

$\square 6.74 \% 18$ Hydrolase

- $3.75 \% 10$ Membrane traffic protein

$\square 4.87 \% 13$ Nucleic acid binding

च $8.24 \% 22$ Receptor

$\square 41.95 \% 112$ Other

C

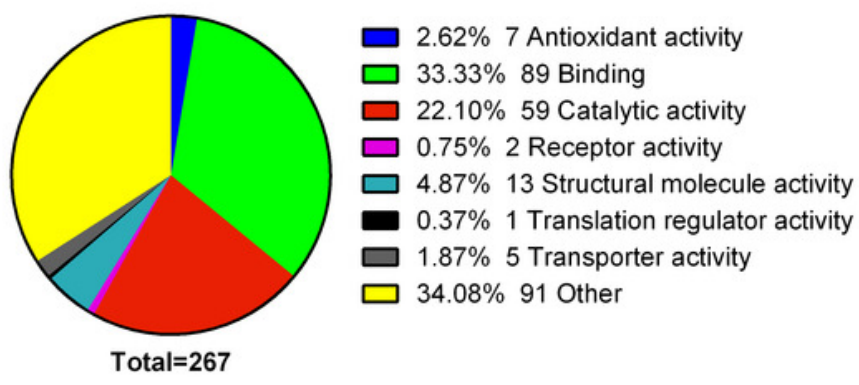




\section{Figure 3}

Molecular function of differentially expressed proteins early.

The figure shows the classification of the 12 proteins with differential expression according to their molecular function. Pathway description.

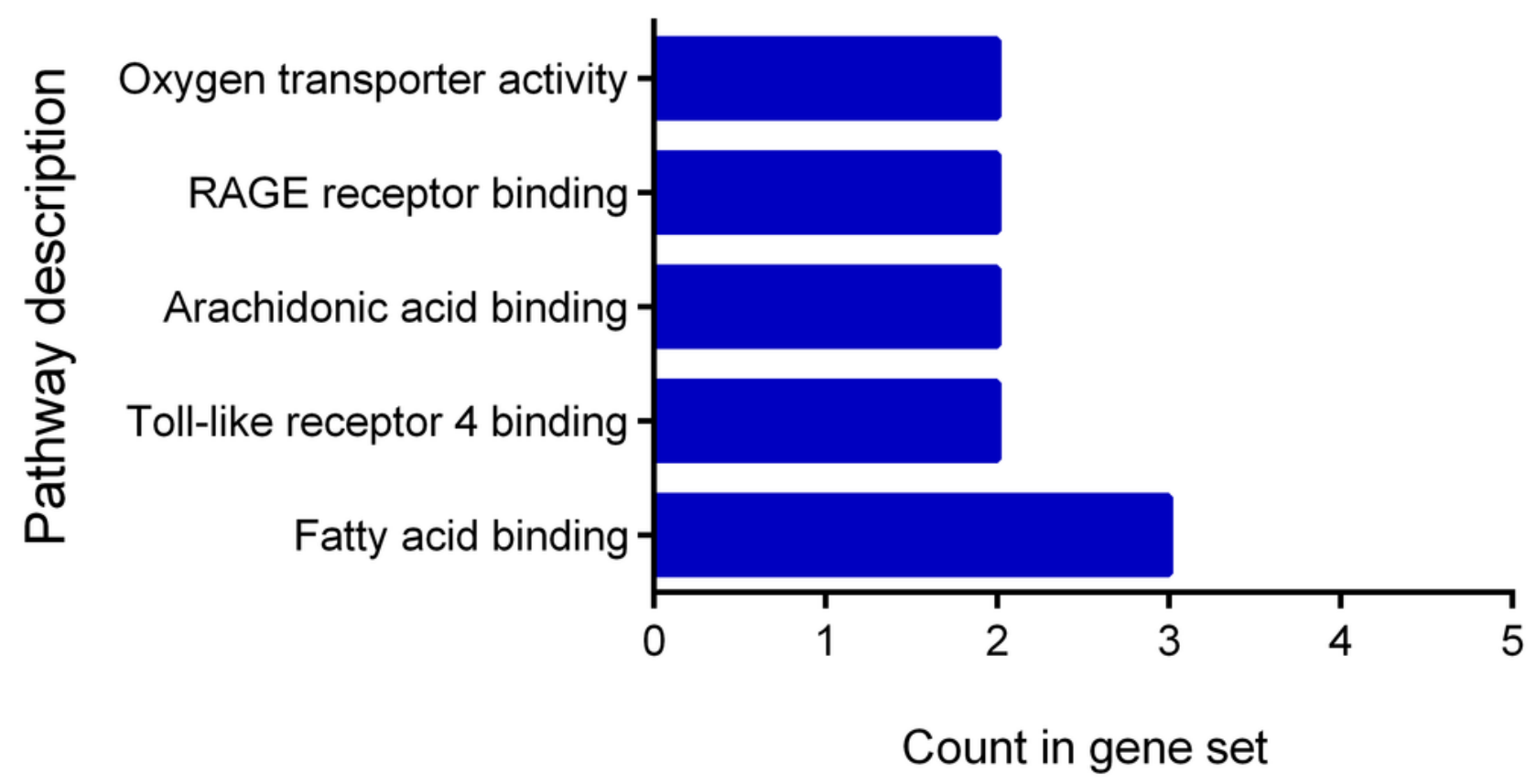




\section{Figure 4}

Interaction network analysis of protein.

Functional interaction network linking the significantly differentially expressed proteins between TD0 and TD7. S100A9: S100 calcium binding protein A9. S100A8: S100 calcium binding protein A8 up-regulated. S100A2: S100 calcium binding protein A2. HBD: Hemoglobin, delta. HBA2: Hemoglobin, alpha 2 down-regulated.

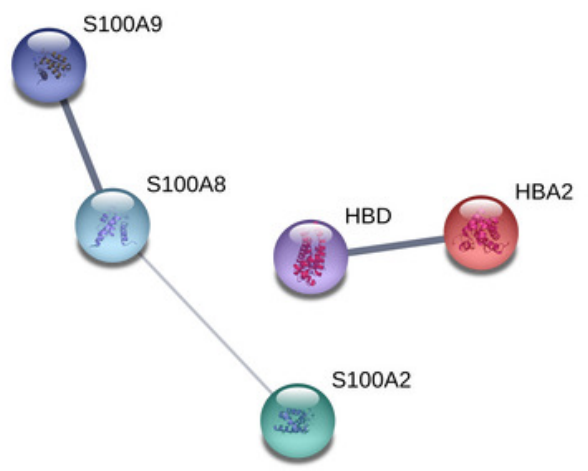


Figure 5

Fold induction of S100A8, S100A9 and SERPINB1 by RT-qPCR.

The figure shows the induction of each gene at day 7 of treatment vs. before treatment calculated by the $\triangle \triangle C T$ method. S100A8 (circles). S100A9 (squares). SERPINB1 (triangle). Each point corresponds to one patient.

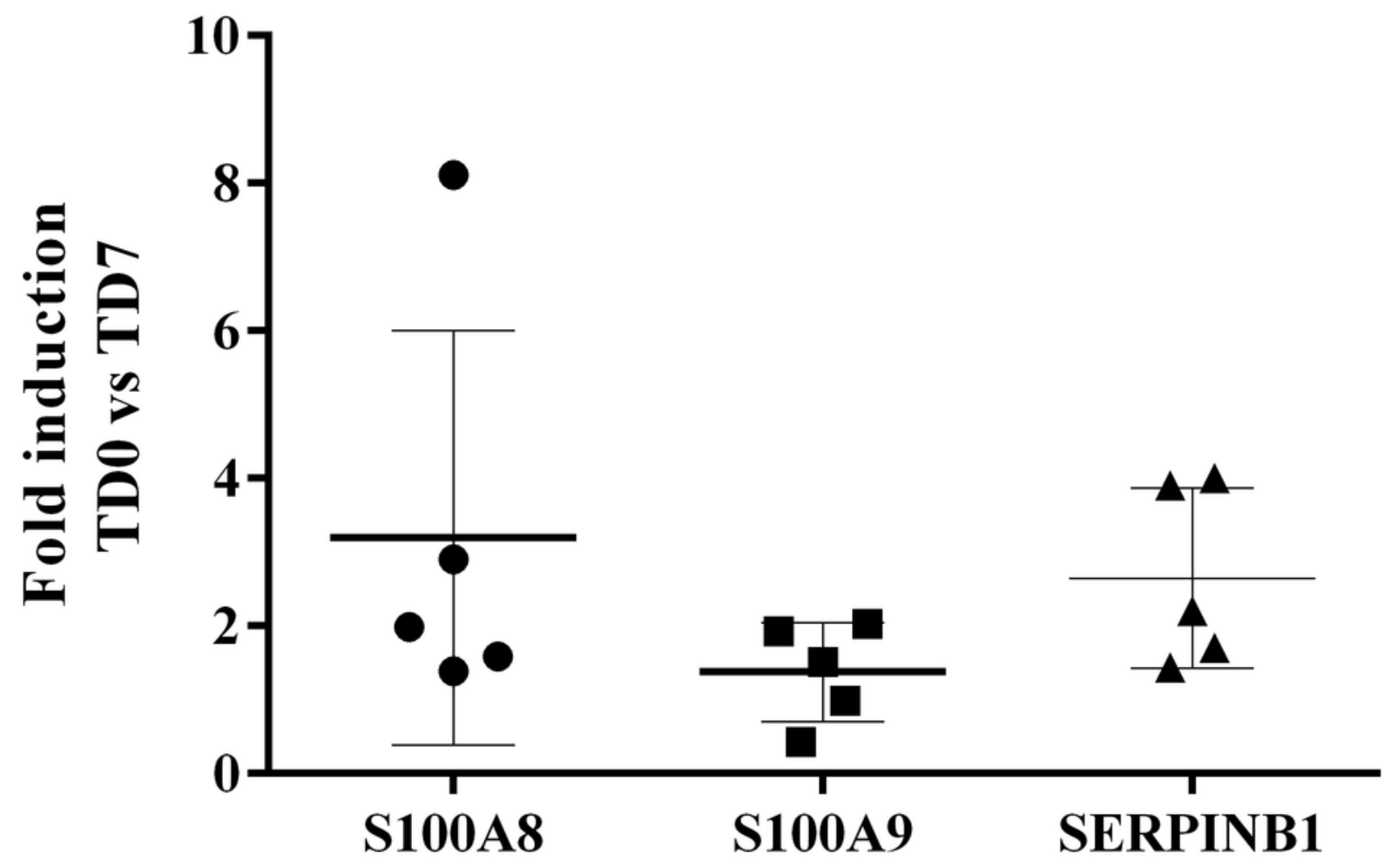

Gene 